\title{
Asparagine attenuates intestinal injury, improves energy status and inhibits AMP-activated protein kinase signalling pathways in weaned piglets challenged with Escherichia coli lipopolysaccharide
}

\author{
Xiuying Wang, Yulan Liu*, Shuang Li, Dingan Pi, Huiling Zhu, Yongqing Hou, Haifeng Shi \\ and Weibo Leng \\ Hubei Key Laboratory of Animal Nutrition and Feed Science, Hubei Collaborative Innovation Center for Animal Nutrition \\ and Feed Safety, Wuhan Polytechnic University, Wuhan 430023, People's Republic of China
}

(Submitted 24 November 2014 - Final revision received 23 April 2015 - Accepted 1 May 2015 - First published online 16 July 2015)

\section{Abstract}

The intestine requires a high amount of energy to maintain its health and function; thus, energy deficits in intestinal mucosa may lead to intestinal damage. Asparagine (Asn) is a precursor for many other amino acids such as aspartate, glutamine and glutamate, which can be used to supply energy to enterocytes. In the present study, we hypothesise that dietary supplementation of Asn could alleviate bacterial lipopolysaccharide (LPS)-induced intestinal injury via improvement of intestinal energy status. A total of twenty-four weaned piglets were assigned to one of four treatments: (1) non-challenged control; (2) LPS + 0\% Asn; (3) LPS + 0.5\% Asn; (4) LPS + 1.0\% Asn. On day 19, piglets were injected with LPS or saline. At $24 \mathrm{~h}$ post-injection, piglets were slaughtered and intestinal samples were collected. Asn supplementation improved intestinal morphology, indicated by higher villus height and villus height:crypt depth ratio, and lower crypt depth. Asn supplementation also increased the ratios of RNA:DNA and protein:DNA as well as disaccharidase activities in intestinal mucosa. In addition, Asn supplementation attenuated bacterial LPS-induced intestinal energy deficits, indicated by increased ATP and adenylate energy charge levels, and decreased AMP:ATP ratio. Moreover, Asn administration increased the activities of key enzymes involved in the tricarboxylic acid cycle, including citrate synthase, isocitrate dehydrogenase and $\alpha$-ketoglutarate dehydrogenase complex. Finally, Asn administration decreased the mRNA abundance of intestinal AMP-activated protein kinase- $\alpha 1$ ( $A M P K \alpha 1)$, $A M P K \alpha 2$, silent information regulator 1 (SIRT1) and PPAR $\gamma$ coactivator-1 $\alpha$ (PGC1 $\alpha$ ), and reduced intestinal AMPK $\alpha$ phosphorylation. Collectively, these results indicate that Asn supplementation alleviates bacterial LPS-induced intestinal injury by modulating the AMPK signalling pathway and improving energy status.

\section{Key words: Asparagine: Intestine: Energy status: Weaned piglets: Lipopolysaccharide}

The intestine is not only important for digestion and absorption of dietary nutrients, but also plays a key role in defence against harmful bacteria-derived endogenous and exogenous agents $^{(1)}$. Many factors such as infection and inflammation can cause intestinal damage and dysfunction ${ }^{(1-3)}$. Recently, research has shown that the intestine requires a high amount of energy to maintain its health and function; thus, energy deficits in intestinal mucosa are closely related to various degrees of injury in the intestine ${ }^{(4)}$. Amino acids can serve as a central fuel source for intestinal mucosa ${ }^{(5)}$. Accumulating evidence suggests that amino acids play a critical role in intestinal integrity, function and health in animals and humans ${ }^{(6)}$.
Thus, nutritional regulation (especially dietary addition of amino acids) targeting intestinal energy metabolism may hold great promise for intestinal disease prevention and improvement of animal and human health.

Asparagine (Asn) is a non-essential amino acid. As has been stated in a recent review, Asn, aspartate, glutamine, glutamate, arginine, citrulline, ornithine and proline are interconvertible via complex interorgan metabolism in most mammals ${ }^{(7)}$. Asn can be converted to aspartate via deamination, and glutamate can subsequently be generated from $\alpha$-ketoglutarate and aspartate by a transamination reaction ${ }^{(8)}$. Emerging evidence has demonstrated that glutamine, aspartate and glutamate

Abbreviations: $\alpha$-KGDHC, $\alpha$-ketoglutarate dehydrogenase complex; AEC, adenylate energy charge; AMPK, AMP-activated protein kinase; Asn, asparagine; BW, body weight; CONTR, non-challenged control group (piglets fed a control diet and injected with $0 \cdot 9 \% \mathrm{NaCl}$ solution); CS, citrate synthase; ICD, isocitrate dehydrogenase; LPS, lipopolysaccharide; pAMPK $\alpha$, phosphorylated AMPK $\alpha$; PGC1 $\alpha$, PPAR $\gamma$ coactivator-1 $\alpha$; SIRT1, silent information regulator 1 ; tAMPK $\alpha$, total AMPK $\alpha$; VCR, villus height:crypt depth ratio.

*Corresponding author: Y. Liu, fax +86 27 83956175, email yulanflower@126.com 
are the major sources of ATP in mammalian enterocytes ${ }^{(5)}$. In addition, Asn contributes to mounting an effective immune response in normal subjects, but can also contribute to abnormal lymphoblastic growth in leukaemia patients ${ }^{(9)}$. Newburg et $a l .{ }^{(10)}$ reported that deletion of Asn from diet formulations led to significant growth deficits in weanling rats. Moreover, Asn stimulates ornithine decarboxylase and cell proliferation in all kinds of cells, including intestinal cells ${ }^{(8,11)}$. However, to our knowledge, only a few studies have focused on the protective effect of Asn in the intestine.

Recent studies have shown that AMP-activated protein kinase (AMPK), silent information regulator 1 (SIRT1) and PPAR $\gamma$ coactivator- $1 \alpha$ (PGC1 $\alpha)$ play key roles in the regulation of cellular energy metabolism ${ }^{(12,13)}$. AMPK is a heterotrimeric serine/threonine kinase. AMPK activation can restore energy status in human cells with mitochondrial dysfunction ${ }^{(14,15)}$. Several lines of evidence indicate that AMPK can increase SIRT1 activity by increasing cellular $\mathrm{NAD}^{+}$levels, culminating in the modulation of downstream target activity ${ }^{(16)}$. In addition, AMPK and SIRT1 have been found to have a direct impact on the activity of PGC- $1 \alpha$ via phosphorylation and deacetylation, respectively ${ }^{(12)}$. These processes can initiate catabolic pathways including fatty acid oxidation and glycolysis to produce ATP, while synchronously inhibiting anabolic processes including fatty acid synthesis and gluconeogenesis to utilise energy ${ }^{(17)}$.

Accordingly, we hypothesise that Asn could improve intestinal integrity by regulating energy status through the modulation of the AMPK signalling pathway. In the present study, we established an acute model of intestinal injury by injecting Escherichia coli lipopolysaccharide (LPS) ${ }^{(3,18)}$. Furthermore, we used a piglet model, which is an excellent animal model for studying the potential nutritional role of Asn in humans ${ }^{(19,20)}$. The aim of the present study was to investigate whether Asn could attenuate negative changes caused by LPS challenge in the intestine, and to elaborate its molecular mechanisms.

\section{Materials and methods}

\section{Animal care and experimental design}

All the experimental procedures were approved by the Animal Care and Use Committee of Hubei Province, China. A total of twenty-four weaned, castrated barrows (Duroc $\times$ Large White $\times$ Landrace, 35 (SEM 1) d old, 8.9 (SEm 0.1) kg initial body weight (BW)) were randomly divided into four treatment groups (six replicate pens per treatment). Piglets were individually caged in a $1.80 \times 1.10 \mathrm{~m}$ pen equipped with a feeder and a nipple drinker to allow ad libitum access to feed and water. All piglets were housed in an environmentally controlled room. The basal diet (Table 1) was formulated to meet $\mathrm{NRC}^{(21)}$ requirements for all nutrients.

The four treatment groups were as follows: (1) nonchallenged control (CONTR) group (piglets fed a control diet and injected with $0.9 \% \mathrm{NaCl}$ solution); (2) LPS + $0 \%$ Asn treatment group (piglets fed the same control diet and injected with E. coli LPS (Escherichia coli serotype 055: B5; Sigma
Chemical, Inc.)); (3) LPS + 0.5\% Asn treatment group (piglets fed a $0.5 \%$ Asn diet and injected with LPS); (4) LPS $+1.0 \%$ Asn treatment group (piglets fed a 1.0\% Asn diet and injected with LPS). The Asn doses (purity >99\%; Amino Acid BioChemical Company Limited) were selected on the basis of our previous studies ${ }^{(22)}$. Our previous investigations showed that before the administration of LPS challenge, dietary supplementation of 0.5 and $1.0 \%$ Asn did not affect growth performance, total and differential leucocyte counts, and serum biochemical parameters of weanling pigs (X Wang, Y Liu, S Li, D Pi, H Zhu, Y Hou, H Shi and W Leng, unpublished results; see online Supplementary Tables S1-S3), indicating that the Asn level of the basal diet was adequate to maintain growth performance and physiological function in weanling pigs under normal physiological conditions. However, our previous studies also showed that after the administration of LPS challenge, dietary supplementation of $0.5 \%$ Asn attenuated weight loss, and both 0.5 and $1.0 \%$ Asn supplementation

Table 1. Ingredients and composition of the experimental diets (as-fed basis)

\begin{tabular}{|c|c|}
\hline Items & Content $(\mathrm{g} / \mathrm{kg})$ \\
\hline \multicolumn{2}{|l|}{ Ingredients } \\
\hline Maize & 570.5 \\
\hline Soyabean meal (44\% CP) & 224 \\
\hline Wheat middling & 50 \\
\hline Fishmeal & 36 \\
\hline Soya protein concentrate & 14 \\
\hline Fat powder* & 20 \\
\hline Milk replacer powder & 30 \\
\hline Limestone & 9.4 \\
\hline Dicalcium phosphate & $12 \cdot 2$ \\
\hline Salt & 3.4 \\
\hline Alat & 13.5 \\
\hline Acidifier $\ddagger$ & $2 \cdot 0$ \\
\hline L-Lys HCl (78.8\% Lys) & $2 \cdot 7$ \\
\hline DL-Met (99\% Met) & 1.0 \\
\hline L-Thr (98\% Thr) & 0.8 \\
\hline Butylated hydroquinone & 0.5 \\
\hline Vitamin and mineral premix§ & 10 \\
\hline \multicolumn{2}{|l|}{ Nutrient composition } \\
\hline Digestible energy\|ף (MJ/kg) & $13 \cdot 6$ \\
\hline Crude protein ${ }^{\star \star}$ & 203 \\
\hline Crude fat ${ }^{\star \star}$ & 44 \\
\hline $\mathrm{Ca}^{\star \star}$ & 8.0 \\
\hline Total $\mathrm{P}^{* *}$ & $7 \cdot 0$ \\
\hline Total Lys & $11 \cdot 1$ \\
\hline Total Met+Cys & 6.5 \\
\hline
\end{tabular}

$\mathrm{CP}$, crude protein.

${ }^{*}$ Rumen-stable fat powder, purchased from Berg + Schmidt.

† In the $0.5 \%$ asparagine diet, $1.35 \%$ alanine was replaced by $0.5 \%$ asparagine, $0.68 \%$ alanine and $0.17 \%$ maize starch. In the $1.0 \%$ asparagine diet, $1.35 \%$ alanine was replaced by $1.0 \%$ asparagine and $0.35 \%$ maize starch. All diets were isonitrogenous.

$\ddagger A$ compound acidifier including lactic acid and phosphoric acid, provided by Wuhan Fanhua Biotechnology Company.

$\S$ Vitamin and mineral premix (defatted rice bran as the carrier) provided the following amounts per $\mathrm{kg}$ of complete diet: retinol acetate, $2700 \mu \mathrm{g}$; cholecalciferol, $62.5 \mu \mathrm{g}$; DL- $\alpha$-tocopheryl acetate, $20 \mathrm{mg}$; menadione, $3 \mathrm{mg}$; vitamin $\mathrm{B}_{12}, 18 \mu \mathrm{g}$; riboflavin, $4 \mathrm{mg}$; niacin, $40 \mathrm{mg}$; pantothenic acid, $15 \mathrm{mg}$; choline chloride, $400 \mathrm{mg}$; folic acid, $700 \mu \mathrm{g}$; thiamin, $1.5 \mathrm{mg}$; pyridoxine, $3 \mathrm{mg}$; biotin, $100 \mu \mathrm{g} ; \mathrm{Zn}, 80 \mathrm{mg}$ $\left(\mathrm{ZnSO}_{4} .7 \mathrm{H}_{2} \mathrm{O}\right) ; \mathrm{Mn}, 20 \mathrm{mg}\left(\mathrm{MnSO}_{4} .5 \mathrm{H}_{2} \mathrm{O}\right) ; \mathrm{Fe}, 83 \mathrm{mg}\left(\mathrm{FeSO}_{4} \cdot \mathrm{H}_{2} \mathrm{O}\right) ; \mathrm{Cu}, 25 \mathrm{mg}$ ( $\left.\mathrm{CuSO}_{4} .5 \mathrm{H}_{2} \mathrm{O}\right) ; \mathrm{I}, 0.48 \mathrm{mg}(\mathrm{KI})$; $\mathrm{Se}, 0.36 \mathrm{mg}\left(\mathrm{Na}_{2} \mathrm{SeO}_{3} .5 \mathrm{H}_{2} \mathrm{O}\right)$.

$\|$ Based on diets containing maize starch.

१ Calculated.

${ }^{\star *}$ Analysed. 
Table 2. Specific primer sequences used for real-time PCR

\begin{tabular}{|c|c|c|c|c|}
\hline Genes & Forward $\left(5^{\prime}-3^{\prime}\right)$ & Reverse $\left(5^{\prime}-3^{\prime}\right)$ & Efficiency (\%) & References \\
\hline AMPK $\alpha 1$ & AAATCGGCCACTACATCCTG & GGATGCCTGAAAAGCTTGAG & 97 & Oliver \& Miles ${ }^{(30)}$ \\
\hline$A M P K \alpha 2$ & AACATGGACGGGTTGAAGAG & CGCAGAAACTCACCATCTGA & 99 & Oliver \& Miles ${ }^{(30)}$ \\
\hline SIRT1 & CTGGAACAGGTTGCAGGAAT & CCTAGGACATCGAGGAACCA & 101 & Weber et al. ${ }^{(31)}$ \\
\hline$P G C 1 \alpha$ & GATGTGTCGCCTTCTTGTTC & CATCCTTTGGGGTCTTTGAG & 99 & Weber et al. ${ }^{(31)}$ \\
\hline$G A P D H$ & CGTCCCTGAGACACGATGGT & GCCTTGACTGTGCCGTGGAAT & 100 & Liu et al. ${ }^{(18)}$ \\
\hline
\end{tabular}

$A M P K \alpha 1 / \alpha 2$, AMP-activated protein kinase- $\alpha 1 / \alpha 2$; SIRT1, silent information regulator $1 ; P G C 1 \alpha$, PPAR $\gamma$ coactivator-1 $\alpha$; GAPDH, glyceraldehyde 3-phosphate dehydrogenase.

attenuated the changes in total and differential leucocyte counts and serum biochemical parameters induced by LPS challenge in weanling pigs ${ }^{(22)}$, indicating the importance of exogenous Asn supply under pathological conditions. Thus, in the present experiment, we focused our investigation upon the effect of 0.5 and $1.0 \%$ dietary Asn supplementation on intestinal variables in LPS-challenged piglets, but did not investigate the effect of Asn in non-LPS-challenged piglets. alanine (purity >99\%; Amino Acid Bio-Chemical Company Limited) to the control, $0.5 \%$ Asn and $1.0 \%$ Asn diets, respectively. Feed consumption and BW were recorded on day 1 and day 19 before the administration of saline or LPS injection. After $19 \mathrm{~d}$ of feeding the control, $0.5 \%$ Asn and 1.0\% Asn diets, the challenged groups were treated with an intraperitoneal injection of LPS at a dose of $100 \mu \mathrm{g} / \mathrm{kg} \mathrm{BW}$, and the nonchallenged group was treated with the same volume of $0.9 \%$ $\mathrm{NaCl}$ solution. The LPS dose was chosen in accordance with our previous experiments ${ }^{(3,18)}$, in which this dose caused acute intestinal injury in weaned pigs. To avoid the potential effects of LPS-induced feed intake reduction on intestinal variables, all piglets were fed the same amount of feed per $\mathrm{kg} \mathrm{BW}$ at $24 \mathrm{~h}$ following the administration of saline or LPS injection, causing no significant difference in feed intake (266, 258, 270 and $276 \mathrm{~g}$, respectively) among the four treatment groups. According to the feed intake of LPS-challenged piglets, the amount of feed per $\mathrm{kg}$ BW was determined at $24 \mathrm{~h}$ after LPS challenge in our preliminary study. Piglets were supplied water ad libitum.

Table 3. Effects of asparagine (Asn) supplementation on serum amino acid concentrations in weaned piglets at $24 \mathrm{~h}$ after the administration of Escherichia coli lipopolysaccharide (LPS) challenge

(Mean values with their pooled standard errors, $n 6$ (one piglet per pen))

\begin{tabular}{|c|c|c|c|c|c|c|c|c|}
\hline \multirow[b]{2}{*}{ Items } & \multicolumn{4}{|c|}{ Treatment } & \multirow[b]{2}{*}{ SEM } & \multicolumn{3}{|c|}{$P^{\star}$} \\
\hline & CONTR & LPS $+0 \%$ Asn & LPS $+0.5 \%$ Asn & LPS $+1.0 \%$ Asn & & CONTR v. LPS + 0\% Asn & Linear & Quadratic \\
\hline Asp $(\mu \mathrm{mol} / \mathrm{l})$ & 64 & 57 & 48 & 87 & 10 & 0.524 & 0.069 & 0.043 \\
\hline Asn $(\mu \mathrm{mol} / \mathrm{l})$ & 82 & 93 & 95 & 137 & 12 & 0.282 & 0.032 & 0.050 \\
\hline Glu ( $\mu \mathrm{mol} / \mathrm{l})$ & 249 & 184 & 148 & 207 & 22 & 0.005 & 0.533 & 0.260 \\
\hline $\operatorname{Gln}(\mu \mathrm{mol} / \mathrm{l})$ & 606 & 394 & 434 & 530 & 31 & 0.003 & $<0.001$ & 0.001 \\
\hline $\operatorname{Arg}(\mu \mathrm{mol} / \mathrm{l})$ & 189 & 270 & 256 & 289 & 37 & 0.099 & 0.735 & 0.839 \\
\hline Cit $(\mu \mathrm{mol} / \mathrm{l})$ & 84 & 88 & 84 & 92 & 4 & 0.608 & 0.459 & 0.442 \\
\hline Orn $(\mu \mathrm{mol} / \mathrm{l})$ & 97 & 82 & 137 & 83 & 14 & 0.463 & 0.960 & 0.013 \\
\hline
\end{tabular}

CONTR, non-challenged control group (piglets fed a control diet and injected with $0.9 \% \mathrm{NaCl}$ solution); Cit, citrulline; Orn, ornithine.

${ }^{*}$ LPS-challenged $(0 \%$ Asn) piglets were compared with CONTR piglets to determine the effects of LPS challenge. Linear and quadratic polynomial contrasts were used to determine the response to Asn supplementation among the LPS-challenged piglets. 

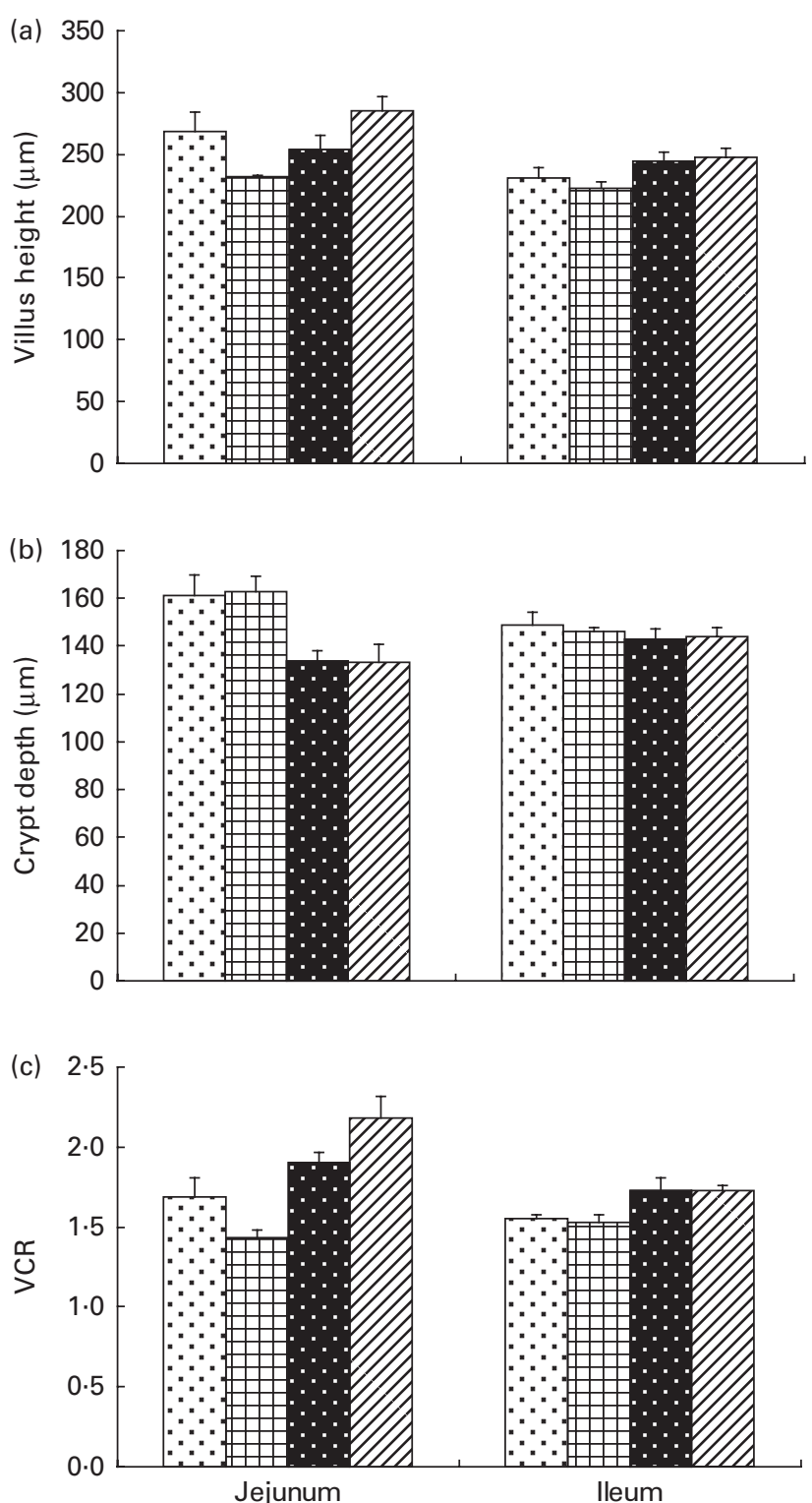

Fig. 1. Effects of asparagine (Asn) supplementation on (a) villus height, (b) crypt depth and (c) villus height:crypt depth ratio (VCR) in weaned piglets at $24 \mathrm{~h}$ after the administration of Escherichia coli lipopolysaccharide (LPS) challenge. Data were analysed as repeated measures with treatments (CONTR (田), LPS + $0 \%$ Asn (田), LPS + $0.5 \%$ Asn (四) and LPS $+1.0 \%$ Asn $(\varpi)$ ) as the between-animal effect and segment (jejunum and ileum) as the within-animal effect. LPS-challenged $(0 \%$ Asn) piglets were compared with CONTR piglets to determine the effects of LPS challenge. Linear (L) and quadratic $(\mathrm{Q})$ polynomial contrasts were used to determine the response to Asn supplementation among the LPS-challenged piglets. Values are means ( $n 6$; one piglet per pen), with their standard errors represented by vertical bars. Villus height $(P<0.001)$ and VCR $(P=0.016)$ in the jejunum were higher than those in the ileum. There were significant treatment $X$ segment interactions observed for crypt depth $(P=0.046)$ and VCR $(P=0.044)$. There were no significant treatment $\times$ segment interactions observed for villus height $(P=0.118)$. (a) CONTR $v$. LPS $+0 \%$ Asn, $P=0.089 ; \mathrm{L}, P<0.001 ; \mathrm{Q}, P=0.001$. (b) Jejunum: CONTR v. LPS $+0 \%$ Asn, $P=0.817$; L, $P=0.002 ; \mathrm{Q}, P=0.006$. lleum: CONTR v. LPS $+0 \%$ Asn, $P=0.602$; L, $P=0.515 ; \mathrm{Q}, P=0.750$. (c) Jejunum: CONTR $v$. LPS $+0 \%$ Asn, $P=0.088 ; L, P<0.001 ; Q, P<0.001$. lleum: CONTR v. LPS $+0 \%$ Asn, $P=0.662 ; \mathrm{L}, P=0.011 ; \mathrm{Q}, P=0.036$. CONTR, non-challenged control group (piglets fed a control diet and injected with $0.9 \% \mathrm{NaCl}$ solution).

\section{Intestinal morphology}

After fixation for $24 \mathrm{~h}$, intestinal samples were dehydrated, embedded in paraffin, sectioned, and stained with haematoxylin and eosin ${ }^{(25)}$. Villus height and crypt depth were measured according to the methods described in our previous study ${ }^{(25)}$.

\section{Intestinal mucosal protein, DNA and RNA contents}

Frozen mucosal samples were homogenised in ice-cold $\mathrm{NaCl}$ solution at a 1:10 (w/v) ratio, followed by centrifugation at $2500 \mathrm{rpm}$ for $10 \mathrm{~min}$ at $4^{\circ} \mathrm{C}$ to collect the supernatant. The supernatant was used for the measurement of protein, RNA and DNA contents. Intestinal mucosal protein content was measured according to the method of Lowry et al. ${ }^{(26)}$. DNA content was measured by a fluorometric assay ${ }^{(27)}$. RNA content was measured by spectrophotometry with a modified Schmidt-Tannhauser method ${ }^{(28)}$.

\section{Intestinal mucosal disaccharidase activities}

Disaccharidase activities in the supernatant of intestinal mucosa were determined according to the methods described by Liu et al. ${ }^{(29)}$ using glucose kits (\#A082-1 for lactase, \#A082-2 for sucrase and \#A082-3 for maltase; Nanjing Jiancheng Bioengineering Institute). In brief, $10 \mu \mathrm{l}$ double-distilled water, glucose standard solution $(5.55 \mathrm{mmol} / \mathrm{l})$ or test samples were added to a test-tube and incubated with $20 \mu \mathrm{l}$ of respective substrate for $20 \mathrm{~min}$ at $37^{\circ} \mathrm{C}$. Then, $10 \mu \mathrm{l}$ of terminating agent and $1000 \mu \mathrm{l}$ of chromogenic agent were added and incubated at $37^{\circ} \mathrm{C}$ for $15 \mathrm{~min}$. Double-distilled water was used to set zero at $505 \mathrm{~nm}$, followed by the reading of the optical density value of each tube. One unit (U) of enzyme activity was defined as $1 \mathrm{nmol}$ substrate hydrolysed/min under assay conditions $\left(37^{\circ} \mathrm{C}, \mathrm{pH} 6 \cdot 0\right)$.

\section{Intestinal mucosal ATP, ADP and AMP concentrations}

Frozen intestinal samples $(0 \cdot 10-0 \cdot 20 \mathrm{~g})$ were homogenised in $2 \mathrm{ml}$ of pre-cooled $1.5 \mathrm{M}$-perchloric acid. The homogenates were centrifuged at $3000 \mathrm{~g}$ for $5 \mathrm{~min}$ at $4^{\circ} \mathrm{C}$, and then the supernatants were collected. A volume of $1 \mathrm{ml}$ supernatant was neutralised with $0.4 \mathrm{ml}$ of $2 \mathrm{M}$-potassium carbonate, followed by centrifugation at $3000 \mathrm{~g}$ for $5 \mathrm{~min}$ at $4^{\circ} \mathrm{C}$. The supernatant was stored at $-80^{\circ} \mathrm{C}$ until analysis. ATP, ADP and AMP concentrations were measured using HPLC, according to the method proposed by Hou et al. ${ }^{(23)}$. Total adenine nucleotide and adenylate energy charge (AEC) levels were calculated by the following equations ${ }^{(23)}$ :

$$
\begin{aligned}
& \mathrm{TAN}=\mathrm{ATP}+\mathrm{ADP}+\mathrm{AMP}, \\
& \mathrm{AEC}=(\mathrm{ATP}+0.5 \mathrm{ADP}) /(\mathrm{ATP}+\mathrm{ADP}+\mathrm{AMP}) .
\end{aligned}
$$

Key enzyme activities of the tricarboxylic acid cycle in intestinal mucosa

The activities of key enzymes including citrate synthase (CS), isocitrate dehydrogenase (ICD) and $\alpha$-ketoglutarate 
dehydrogenase complex $(\alpha-\mathrm{KGDHC})$ involved in the tricarboxylic acid cycle were assayed according to commercial enzyme assay kits (\#45126 for CS, \#45 234 for ICD and \#45 157 for $\alpha$-KGDHC; Shanghai Yuanye Biotechnology Company). All variables were measured according to the manufacturer's guidelines. Briefly, $50 \mu \mathrm{l}$ of standard solutions or diluted intestinal mucosal supernatants were added to a separately identified well of the microelisa stripplate. A solution of $100 \mu \mathrm{l}$ horseradish peroxidase (HRP) conjugate reagent was added to each well, and then covered with an adhesive strip and incubated for $60 \mathrm{~min}$ at $37^{\circ} \mathrm{C}$. After incubation, the plates were washed for five times with wash solutions. Subsequently, $50 \mu \mathrm{l}$ of chromogen solution A and $50 \mu \mathrm{l}$ of chromogen solution B were added, followed by incubation for $15 \mathrm{~min}$ at $37^{\circ} \mathrm{C}$. Then, $50 \mu \mathrm{l}$ of stop solution were added. Optical density was read at $450 \mathrm{~nm}$ using an ELISA plate reader (Model 550; Bio-Rad) within $15 \mathrm{~min}$. The activities of the key enzymes in the tricarboxylic acid cycle were determined by comparing the optical density of intestinal samples with the standard curve. Results for CS and ICD activities were expressed as $\mu \mathrm{IU} / \mathrm{mg}$ protein. One IU/mg protein was defined as $1 \mu \mathrm{mol}$ substrate hydrolysed/min per mg protein under specified assay conditions.

\section{mRNA abundance analysis by real-time $P C R$}

Total RNA was extracted from intestinal mucosa using TRIzol reagent (\#9108; TaKaRa Biotechnology (Dalian) Company Limited) following the manufacturer's instructions. RNA was spectrophotometrically quantified by determining absorbance at $260 \mathrm{~nm}$, and integrity was assessed by agarose gel electrophoresis. Both genomic DNA removal and complementary DNA synthesis were performed using a PrimeScript RT reagent kit with a gDNA eraser (\#RR047A; TaKaRa Biotechnology (Dalian) Company Limited) according to the protocol of the manufacturer. Real-time PCR analysis for gene expression was carried out on the Applied Biosystems 7500 Real-Time PCR System (Applied Biosystems, Life Technologies) using a SYBR $^{\circledR}$ Premix Ex Taq ${ }^{\text {TM }}$ (Tli RNase H Plus) qPCR kit (\#RR420A; TaKaRa Biotechnology (Dalian) Company Limited), according to the manufacturer's guidelines. The PCR programme was as follows: $95^{\circ} \mathrm{C}$ for $30 \mathrm{~s}$, followed by forty cycles of $95^{\circ} \mathrm{C}$ for $5 \mathrm{~s}$ and $60^{\circ} \mathrm{C}$ for $34 \mathrm{~s}$. The primer pairs used are presented in Table 2. The sequences of the PCR primers were according to previous studies ${ }^{(18,30,31)}$. Quantitative PCR efficiencies of these primers used were close to $100 \%$ in the present experiment. The PCR products of different primers were verified by agarose gel electrophoresis and sequencing. The expression of the target genes $v$. housekeeping gene (glyceraldehyde 3-phosphate dehydrogenase, GAPDH) was determined by the formula $2^{-\Delta \Delta C_{\mathrm{T}}}$ of Livak \& Schmittgen ${ }^{(32)}$. The results of the present study suggest that there was no difference in the expression of GAPDH among the tissues and treatments. The relative mRNA abundance of each target gene was normalised to the control group.

\section{Protein abundance analysis by Western blot}

Protein immunoblot analysis was carried out in accordance with the previously described method ${ }^{(18)}$. Briefly, intestinal 
samples $(0 \cdot 15-0 \cdot 20 \mathrm{~g})$ were homogenised in $1 \mathrm{ml}$ of lysis buffer containing protease inhibitors, and centrifuged at $12000 \mathrm{~g}$ for $15 \mathrm{~min}$ at $4^{\circ} \mathrm{C}$ to collect supernatants for Western blot and protein assay. The protein contents of the supernatants were determined using the bicinchoninic acid reagent ${ }^{(18,33)}$. Equal amounts of intestinal mucosal proteins were loaded onto $10 \%$ polyacrylamide gels, separated through SDSPAGE, and then transferred to blotting membranes. Immunoblots were blocked with $3 \%$ bovine serum albumin in TBS (Tris-HCl-buffered saline, including Tris- $\mathrm{HCl}, \mathrm{NaCl}$ and $\mathrm{KCl}$ )/ Tween-20 buffer for $60 \mathrm{~min}$ at room temperature. Then, the membranes were incubated overnight at $4^{\circ} \mathrm{C}$ with primary antibodies, followed by incubation with a secondary antibody for $120 \mathrm{~min}$ at room temperature. Specific primary antibodies included rabbit anti-phosphorylated AMPK $\alpha$ ( $\mathrm{pAMPK} \alpha$, Thr172, 1:1000, \#2535; Cell Signaling Technology, Inc.), rabbit anti-total AMPK $\alpha$ (tAMPK $\alpha, 1: 1000, \# 2532$; Cell Signaling Technology, Inc.) and mouse anti- $\beta$-actin (1:10 000, \#A2228; SigmaAldrich, Inc.). Secondary antibodies included goat anti-rabbit IgG HRP (1:5000, \#ANT020; Antgene Biotech) and goat antimouse IgG HRP (1:5000, \#ANT019; Antgene Biotech). In our previous study, these antibodies were validated in weanling pigs ${ }^{(33)}$. Blots were developed using an enhanced chemiluminescence Western blotting kit (Amersham Biosciences), and visualised using a Gene Genome Bioimaging System (Alpha Innotech). Bands were analysed by densitometry using GeneTools software (Syngene). pAMPK $\alpha$ was normalised to the total protein content of AMPK $\alpha$.

\section{Statistical analysis}

Experimental data were analysed by variance specific for repeated measures using the mixed procedure of SAS (SAS Institute, Inc.), with treatments as the between-animal effect and gut segment (jejunum and ileum) as the within-animal effect according to the following model:

$$
Y_{\mathrm{ijk}}=\mu+\alpha_{\mathrm{i}}+\omega_{\mathrm{j}}+(\alpha \omega)_{\mathrm{ij}}+\mu_{\mathrm{k}}+\varepsilon_{\mathrm{ijk}},
$$

where $\alpha_{\mathrm{i}}$ is the effect of the treatment ( $\mathrm{i}=$ CONTR, LPS $+0 \%$ Asn, LPS $+0.5 \%$ Asn and LPS $+1.0 \%$ Asn); $\omega_{\mathrm{j}}$ is the segment (jejunum and ileum); $\alpha \omega_{\mathrm{ij}}$ is the interaction between treatment and segment; $\mu_{\mathrm{k}} \sim N\left(0, \tau^{2}\right)$ accounts for repeated measures made on the same individual, thereby rendering these observations correlated. The error term $\varepsilon_{\mathrm{ijk}} \sim N\left(0, \sigma^{2}\right)$ represents unexplained variation. The variance interaction between segment and diet was described as random by using

$$
\alpha \omega_{\mathrm{ij}} \approx N\left(0, \varpi^{2}\right)(\text { type }=\operatorname{arh}(1)) .
$$

When a significant interaction between treatment and segment occurred, comparisons were made among the treatments in each segment (jejunum or ileum). LPS-challenged piglets ( $0 \%$ Asn) were compared with CONTR piglets to determine the effect of LPS challenge. Linear and quadratic polynomial contrasts were used to determine the response to dietary Asn supplementation among the LPS-challenged piglets. Results are expressed as means with their pooled standard errors. $P \leq 0.05$ was considered as statistically significant, and $0.05<P<0.10$ indicated a trend. 


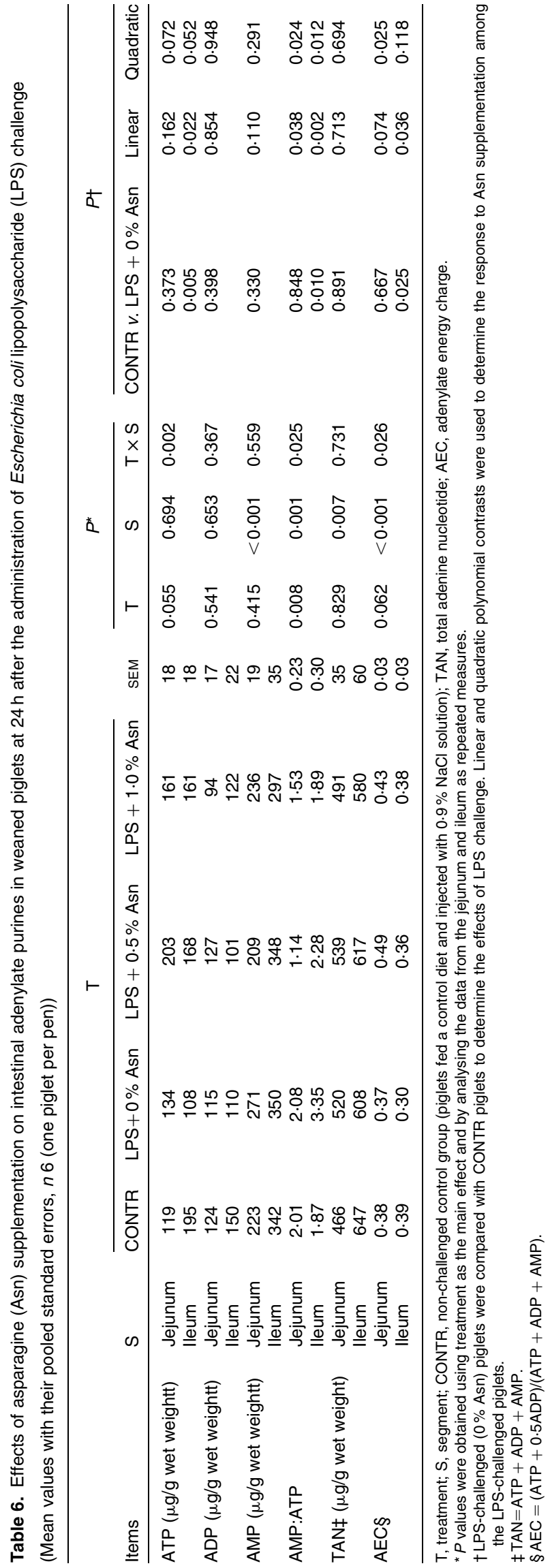

\section{Results \\ Growth performance}

During the entire $19 \mathrm{~d}$ feeding trial (pre-challenge), there were no differences in initial $\mathrm{BW}(9 \cdot 0,8 \cdot 8,9 \cdot 0$ and $8 \cdot 8 \mathrm{~kg}$, respectively) and final $\mathrm{BW}(18.0,17.5,18.3$ and $18.7 \mathrm{~kg}$, respectively), average daily gain $(477,459,492$ and $524 \mathrm{~g}$, respectively), average daily feed intake (733, 741, 703 and $759 \mathrm{~g}$, respectively) and feed:gain ratio $(1.54,1.64,1.43$ and $1 \cdot 44$, respectively) among the four treatment groups.

\section{Serum amino acid concentrations}

Compared with CONTR piglets, LPS-challenged ( $0 \%$ Asn) piglets had decreased glutamate and glutamine concentrations $(P<0.05$; Table 3$)$. Among the LPS-challenged piglets, Asn supplementation increased the concentrations of aspartate (quadratic, $P<0.05$ ), Asn (linear, $P<0.05$; quadratic, $P \leq 0.05$ ), glutamine (linear, $P<0.001$; quadratic, $P<0.05$ ) and ornithine (quadratic, $P<0 \cdot 05$ ).

\section{Intestinal morphology}

LPS challenge ( $0 \%$ Asn) caused fever, diarrhoea, anorexia, shivering and inactivity within $1 \mathrm{~h}$ in all piglets (data not shown). Villus height and villus height:crypt depth ratio (VCR) in the jejunum were higher than those in the ileum $(P<0.05$; Fig. 1). No significant treatment $\times$ segment interaction was observed for villus height. Overall, compared with CONTR piglets, LPS-challenged ( $0 \%$ Asn) piglets tended to have decreased villus height $(P=0 \cdot 089)$. Among the LPS-challenged piglets, Asn supplementation increased villus height (linear, $P<0 \cdot 001$; quadratic, $P<0 \cdot 05$ ).

Significant treatment $x$ segment interactions were observed for crypt depth and VCR $(P<0 \cdot 05)$. Compared with CONTR piglets, LPS-challenged ( $0 \%$ Asn) piglets tended to have decreased VCR in the jejunum $(P=0.088)$. Among the LPS-challenged piglets, Asn supplementation increased the VCR in the jejunum and ileum (linear, $P<0.05$; quadratic, $P<0.05$ ), and decreased crypt depth in the jejunum (linear, $P<0 \cdot 05$; quadratic, $P<0 \cdot 05$ ).

\section{Protein, DNA and RNA contents}

The RNA:DNA ratio in the ileum was higher than that in the jejunum $(P<0.05$; Table 4$)$. There were significant treatment $X$ segment interactions observed for RNA:DNA and protein:DNA ratios $(P<0.05)$, and a trend for treatment $\times$ segment interactions was observed for mucosal protein content $(P=0.069)$. Relative to CONTR piglets, LPS-challenged ( $0 \%$ Asn) piglets had decreased mucosal protein content in the jejunum and ileum $(P<0.05)$, and RNA:DNA and protein:DNA ratios in the ileum $(P<0 \cdot 05)$. Among the LPS-challenged piglets, Asn supplementation increased the RNA:DNA and protein:DNA ratios in the jejunum and ileum (linear, $P<0 \cdot 05$; quadratic, $P \leq 0 \cdot 05$ ). 


\section{Disaccharidase activities}

Disaccharidase activities in the jejunum were higher than those in the ileum $(P<0.001$; Table 5). No significant treatment $\times$ segment interaction was found for lactase, maltase and sucrase activities. Overall, compared with CONTR piglets, LPS-challenged ( $0 \%$ Asn) piglets had decreased sucrase activity $(P \leq 0 \cdot 05)$. Among the LPS-challenged piglets, Asn supplementation increased lactase activity (linear, $P<0.05$; quadratic, $P<0 \cdot 05$ ).

\section{ATP, ADP and AMP concentrations in intestinal mucosa}

AMP concentrations, AMP:ATP ratios and total adenine nucleotide concentrations in the jejunum were lower than those in the ileum, and AEC levels in the jejunum were higher than those in the ileum $(P<0 \cdot 05$; Table 6). Significant treatment $\times$ segment interactions were observed for ATP concentrations, AMP:ATP ratios and AEC levels $(P<0 \cdot 05)$. Compared with CONTR piglets, LPS-challenged ( $0 \%$ Asn) piglets had decreased ATP concentrations and AEC levels, and increased AMP:ATP ratios in the ileum $(P<0.05)$. Among the LPS-challenged piglets, Asn supplementation increased ATP concentrations in the ileum (linear, $P<0.05$ ) and AEC levels in the jejunum (quadratic, $P<0.05$ ) and ileum (linear, $P<0.05$ ), but decreased AMP:ATP ratios in the jejunum (linear, $P<0.05$; quadratic, $P<0.05$ ) and ileum (linear, $P<0.05$; quadratic, $P<0 \cdot 05$ ).

\section{Key enzyme activities of the tricarboxylic acid cycle}

The activities of CS and ICD in the jejunum were higher than those in the ileum $(P<0 \cdot 001)$, and $\alpha$-KGDHC activity in the jejunum was lower than that in the ileum $(P<0 \cdot 05)$ (Table 7). No significant treatment $\times$ segment interaction was found for the activities of CS and $\alpha$-KGDHC. Overall, compared with CONTR piglets, LPS-challenged ( $0 \%$ Asn) piglets had decreased CS and $\alpha$-KGDHC activities $(P<0 \cdot 05)$. Among the LPS-challenged piglets, Asn supplementation increased the activities of CS and $\alpha$-KGDHC (quadratic, $P<0 \cdot 05$ ).

A significant treatment $\times$ segment interaction was observed for ICD activity $(P<0.05)$. Compared with CONTR piglets, LPS-challenged ( $0 \%$ Asn) piglets had decreased ileal ICD activity $(P<0.05)$. Among the LPS-challenged piglets, Asn supplementation increased jejunal (quadratic, $P<0 \cdot 001$ ) and ileal (linear, $P<0 \cdot 001$; quadratic, $P<0 \cdot 001$ ) ICD activity.

mRNA expression of AMP-activated protein kinase $\alpha 1$, $A M P$-activated protein kinase $\alpha 2$, silent information regulator 1 and PPAR $\gamma$ coactivator- $1 \alpha$

The mRNA abundance of $A M P K \alpha 1$ and $A M P K \alpha 2$ in the jejunum was lower than that in the ileum $(P<0.05$; Table 8$)$. There was a significant treatment $\times$ segment interaction observed for the mRNA abundance of AMPK $\alpha 2(P<0.05)$, and a trend for the treatment $\times$ segment interaction observed for the mRNA abundance of $A M P K \alpha 1(P=0.084)$. Compared with CONTR piglets, LPS-challenged ( $0 \%$ Asn) piglets had 
increased mRNA abundance of ileal $A M P K \alpha 1$ and $A M P K \alpha 2$ $(P<0 \cdot 05)$. Among the LPS-challenged piglets, Asn supplementation decreased the mRNA abundance of jejunal and ileal $A M P K \alpha 1$, and ileal $A M P K \alpha 2$ (linear, $P<0.05$; quadratic, $P<0 \cdot 05)$.

No significant treatment $\times$ segment interaction was observed for the mRNA abundance of SIRT1 and PGC1 $\alpha$. Compared with CONTR piglets, LPS-challenged ( $0 \%$ Asn) piglets had increased mRNA abundance of PGC1 $\alpha(P<0.05)$. Among the LPS-challenged piglets, Asn supplementation decreased the mRNA abundance of SIRT1 (linear, $P<0.05$ ) and $P G C 1 \alpha$ (linear, $P<0.05$; quadratic, $P<0.05$ ).

Protein phosphorylation and abundance of AMP-activated protein kinase $\alpha$

The pAMPK $\alpha$ :tAMPK $\alpha$ ratio in the jejunum were lower than that in the ileum $(P<0.05)$, and the protein abundance of tAMPK $\alpha$ in the jejunum was higher than that in the ileum $(P<0.001)$ (Fig. 2). A trend for the treatment $\times$ segment interaction was observed for the pAMPK $\alpha$ :AMPK $\alpha$ ratio $(P=0.073)$. Compared with CONTR piglets, LPS-challenged $(0 \%$ Asn) piglets had increased ileal pAMPK $\alpha$ :tAMPK $\alpha$ ratio $(P<0.05)$. Among the LPS-challenged piglets, Asn supplementation decreased the ileal pAMPK $\alpha$ :tAMPK $\alpha$ ratio (linear, $P<0.05$; quadratic, $P<0.05$ ).

\section{Discussion}

LPS is the main constituent of the outer membrane of Gramnegative bacteria ${ }^{(3)}$. Increasing evidence indicates that LPS is a cofactor in intestinal injury ${ }^{(3,18)}$. Intestinal alterations including morphological injury, increased mucosal permeability and bacterial translocation have often been reported after intraperitoneal injection of $\operatorname{LPS}^{(3)}$. The pathogenesis of LPS-induced intestinal injury is viewed as a complex event, and it has been correlated with increased inflammation caused by LPS challenge that leads to the expression of pro-inflammatory cytokines $^{(34,35)}$. In addition, LPS is known to cause significant damage to gastrointestinal oxygen metabolism and mitochondria dysfunction, leading to decreased ATP concentrations and eventually intestinal damage ${ }^{(36)}$. Our previous studies have suggested that dietary supplementation with $0.5 \%$ Asn alleviated growth suppression, and both 0.5 and $1.0 \%$ dietary Asn supplementation attenuated the changes in total and differential leucocyte counts and serum biochemical parameters in weaned piglets after the administration of LPS challenge ${ }^{(22)}$. Therefore, we extended the finding to the intestine to explore the effect of Asn supplementation on intestinal injury. To our knowledge, this is the first study to evaluate whether dietary Asn supplementation could attenuate intestinal injury in weanling piglets challenged with LPS.

Villus height, crypt depth and VCR were used to measure intestinal morphology ${ }^{(18)}$. Mucosal protein contents, RNA:DNA and protein:DNA ratios are important biochemical indices for intestinal development ${ }^{(3)}$. Mucosal disaccharidases, namely lactase, maltase and sucrase, are directly involved in the energy supply of organism, and can mirror intestinal 

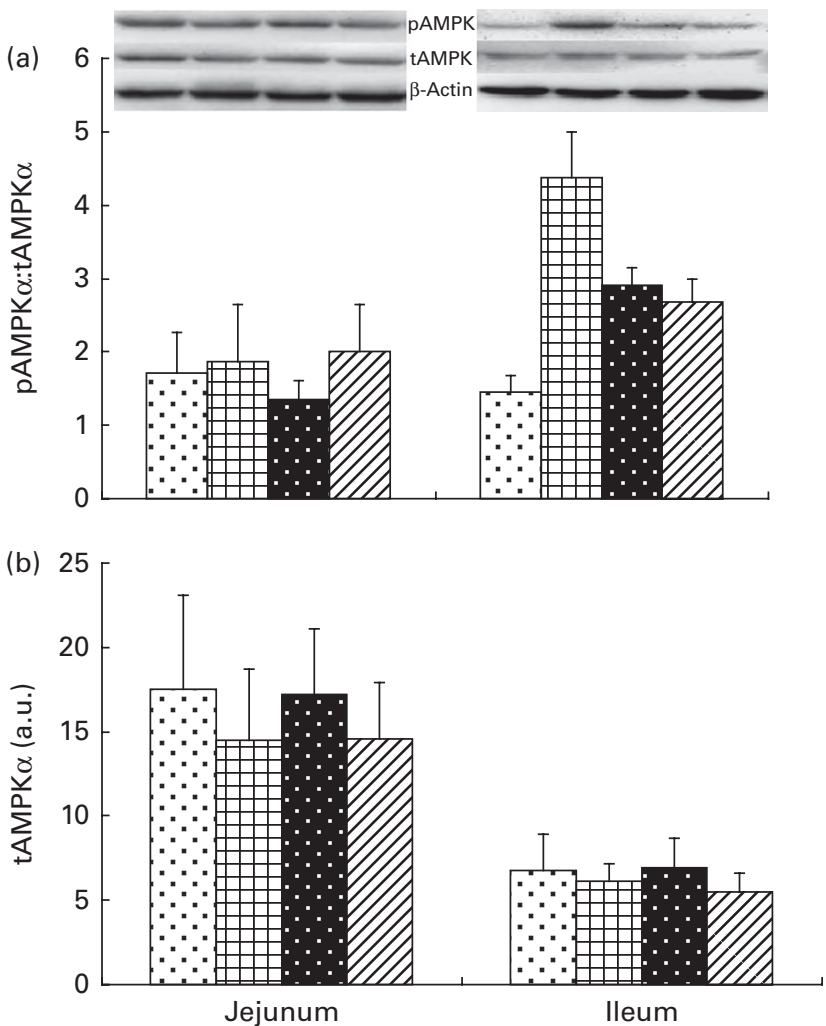

Fig. 2. Effects of asparagine (Asn) supplementation on the (a) phosphorylated AMP-activated protein kinase (pAMPK $\alpha$ ):total AMP-activated protein kinase (tAMPK $\alpha$ ) ratio and (b) protein abundance of tAMPK $\alpha$ in weaned piglets at $24 \mathrm{~h}$ after the administration of Escherichia coli lipopolysaccharide (LPS) challenge. The bands shown are the representative Western blot images of pAMPK $\alpha(62 \mathrm{kDa})$, tAMPK $\alpha(62 \mathrm{kDa})$ and $\beta$-actin $(42 \mathrm{kDa}) . \beta$-Actin was from the same blot as the proteins of interest. Data were analysed as repeated measures with treatments (CONTR (曰), LPS + $0 \%$ Asn ( $\boxplus$ ), LPS $+0.5 \%$ Asn (四) and LPS $+1.0 \%$ Asn (区) ) as the between-animal effect and segment (jejunum and ileum) as the within-animal effect. LPSchallenged $(0 \%$ Asn) piglets were compared with CONTR piglets to determine the effects of LPS challenge. Linear (L) and quadratic (Q) polynomial contrasts were used to determine the response to Asn supplementation among the LPS-challenged piglets. Values are means ( $n 6$; one piglet per pen), with their standard errors represented by vertical bars. a.u., Arbitrary units. The pAMPK $\alpha$ :AMPK $\alpha$ ratio in the jejunum was lower than that in the ileum $(P=0.006)$, and protein abundance of tAMPK $\alpha$ in the jejunum was higher than that in the ileum $(P<0.001)$. A trend for the treatment $\times$ segment interaction was observed for the pAMPK $\alpha$ :tAMPK $\alpha$ ratio $(P=0.073)$. There was no significant treatment $\times$ segment interaction observed for protein abundance of tAMPK $\alpha(P=0.947)$. (a) Jejunum: CONTR $v$. LPS $+0 \%$ Asn, $P=0.879 ; \quad L, P=0.957 ; Q, P=0.722$. lleum: CONTR $v$. LPS $+0 \%$ Asn, $P=0.001 ; \quad L, P=0.007 ; \quad Q, P=0.028$. (b) CONTR $v$. LPS $+0 \%$ Asn, $P=0.688 ; L, P=0.917 ; Q, P=0.834$. CONTR, non-challenged control group (piglets fed a control diet and injected with $0.9 \% \mathrm{NaCl}$ solution).

digestive function ${ }^{(37)}$. In the present study, villus height and disaccharidase activities in the jejunum were higher than those in the ileum, which is similar to the report of Rubio et al. ${ }^{(38)}$. LPS challenge decreased villus height, VCR, mucosal protein content, RNA:DNA and protein:DNA ratios, and disaccharidase activities, which is consistent with the findings of Liu et $a l^{(3,29)}$. These data indicate that injection of LPS caused intestinal injury in weaned pigs. Asn supplementation to the LPS-challenged pigs increased villus height and VCR, decreased crypt depth, increased RNA:DNA and protein:DNA ratios, and increased disaccharidase activities linearly and quadratically. These data indicate that Asn protected the intestine from damage. Until now, little is known about the nutritional significance of Asn in the intestine. Previous studies have found that Asn stimulates enterocyte proliferation in the small intestine of pigs ${ }^{(8,11)}$. However, the protective mechanism of Asn remains unknown. Rhoads et al. ${ }^{(8)}$ reported that Asn can be converted to aspartate, and glutamate can subsequently be generated from $\alpha$-ketoglutarate and aspartate. In the present study, Asn supplementation to the LPS-challenged pigs increased serum concentrations of aspartate, Asn, glutamine and ornithine linearly and quadratically. In this way, it is possible that Asn may be a precursor for many other amino acids to be produced on demand to meet the requirements of enterocytes.

The intestine takes up a high amount of energy to sustain its integrity, function and health ${ }^{(39,40)}$, and energy deficits in intestinal mucosa may relate to intestinal injury ${ }^{(23)}$. Most cellular processes need energy and are driven directly or indirectly by hydrolysing ATP to ADP and phosphate, or less frequently to AMP and pyrophosphate. The AMP:ATP ratio is a sensitive indicator of cellular energy state ${ }^{(41)}$. In comparison with the level of a single nucleotide, the energy charge of the adenyl pool is a better way to measure the energy status of a tissue $^{(23)}$. In the present study, LPS challenge decreased ATP concentrations and AEC levels, and increased AMP:ATP ratios. Similarly, Hou et al. ${ }^{(23)}$ reported that LPS challenge altered the cellular energy status in intestinal mucosa. Asn supplementation to the LPS-challenged pigs increased ATP concentrations linearly and AEC levels linearly and quadratically, and decreased AMP:ATP ratios linearly and quadratically. These data support the notion that Asn supplementation attenuated LPS-induced intestinal damage possibly via modulating the adenine nucleotide pool.

The tricarboxylic acid cycle is a central route for energy production in the intestine ${ }^{(42)}$. Key enzymes involved in the tricarboxylic acid cycle include CS, ICD and $\alpha$-KGDHC. The enzyme CS catalyses the first step of the tricarboxylic acid cycle by taking molecules of acetate and attaching them to oxaloacetate $^{(43)}$. ICD is responsible for catalysing the oxidative decarboxylation of isocitrate into $\alpha$-ketoglutarate and $\mathrm{CO}_{2}^{(44)}$. $\alpha$-KGDHC is a multi-enzymatic complex that converts $\alpha$-ketoglutarate to succinyl-CoA ${ }^{(45)}$. In the present study, Asn supplementation to the LPS-challenged pigs attenuated the decrease in the activities of the key enzymes in the tricarboxylic acid cycle linearly and quadratically. This may due to the conversion of Asn to aspartate ${ }^{(8)}$, which can be converted to tricarboxylic acid cycle intermediates (such as oxaloacetate $)^{(46)}$. In the present study, it is possible that dietary supplementation with Asn improved intestinal energy status by enhancing the key enzyme activities of the tricarboxylic acid cycle.

AMPK is an energy regulator whose primary role involves maintaining the intracellular energy balance in eukaryotic evolution $^{(12)}$. It can be activated by mechanisms including phosphorylation upon allosteric activation by increasing the AMP:ATP ratio ${ }^{(47)}$. To restore the cellular energy status, the activation of AMPK can switch on ATP-producing processes 
while synchronously switching off ATP-consuming processes $^{(47)}$. In addition, AMPK could chronically promote cellular ability to produce ATP and diminish potentially adverse cellular events ${ }^{(48)}$. Several reports have revealed that AMPK can enhance the activity of SIRT1 by increasing cellular $\mathrm{NAD}^{+}$levels ${ }^{(16)}$. SIRT1 was found to be a major regulator of muscle adaptation to nutrient availability ${ }^{(49,50)}$. When activated, SIRT1 also enhances mitochondrial oxidative function and leads to selective nutrient utilisation to regulate energy balance $^{(13)}$. Furthermore, SIRT1 deactetylation has been proposed to be a potential activator for the transcriptional activity of PGC1 $\alpha^{(51)}$. D'Errico et al. ${ }^{(52)}$ reported that PGC1 $\alpha$ modulates mitochondrial biogenesis and function. In the present study, LPS challenge increased the mRNA abundance of $A M P K \alpha 1, A M P K \alpha 2$ and $P G C 1 \alpha$, and the phosphorylation of AMPK $\alpha$. In agreement with the results of the present study, Hou et al. ${ }^{(23)}$ reported that LPS challenge increased intestinal AMPK $\alpha$ phosphorylation. Asn supplementation to the LPSchallenged pigs decreased intestinal $A M P K \alpha 1, A M P K \alpha 2$, SIRT1 and PGC1 $\alpha$ mRNA abundance, and decreased ileal AMPK $\alpha$ phosphorylation linearly and quadratically. In the present study, consistent with reduced intestinal AMP:ATP ratios and increased ATP concentrations, dietary Asn supplementation inhibited intestinal AMPK signalling pathway in response to LPS treatment. It is possible that the reduced intestinal AMP:ATP ratios and the increased ATP concentrations in enterocytes might be enough to inhibit the AMPK signalling pathway in LPS-challenged piglets fed the Asn diet. Future research is needed to elucidate the mechanisms for the inhibitory effect of Asn on the AMPK signalling pathway.

In the present study, only two doses of Asn ( 0.5 and $1.0 \%$ Asn) were used. The higher concentration of Asn (1.0\%) had a worse effect on some parameters characteristic for energy status compared with the lower concentration $(0.5 \%)$. We speculate that the different effects of the two doses of Asn might be due to the following mechanisms. First, Asn can be converted to glutamine via complex interorgan metabolism $^{(7)}$. In the present study, Asn supplementation to the LPS-challenged pigs increased serum glutamine concentrations. Holecek ${ }^{(53)}$ reported that enhanced glutamine intake competed with intestinal absorption of a number of amino acids, which might affect amino acid and protein metabolism in the gut, liver and whole body. Second, as with all other nutrients, it is possible that excessive amount of Asn in diets can cause amino acid imbalances and toxicity. In addition, we used an acute model of LPS challenge, which is different from a more chronic LPS challenge situation (e.g. during $8-15 \mathrm{~d}$ post-weaning). Thus, future studies including more Asn doses are needed to better understand the effects of Asn supplementation in an acute or chronic LPS challenge situation. Moreover, the effect of dietary Asn supplementation on some variables differed between the jejunum and the ileum. This might be related to different microenvironments among various segments of the gut at molecular and cellular levels ${ }^{(54)}$.

In summary, dietary supplementation of Asn mitigates intestinal injury and improves intestinal energy status of weaned piglets challenged by LPS. In addition, Asp supplementation modulates intestinal AMPK signalling pathway. These novel findings not only contribute to the understanding of the mode of action of Asn in the intestine of pigs, but also hold great significance for improving infant nutrition.

\section{Supplementary material}

To view supplementary material for this article, please visit http://dx.doi.org/10.1017/S0007114515001877

\section{Acknowledgements}

The present study was supported by the National Natural Science Foundation of China (grant no. 31422053, 31372318 and 31172222) and the Project of Natural Science Foundation of Hubei Province (grant no. 2013CFA029).

The authors' contributions are as follows: Y. L. designed the research; X. W., Y. L., S. L., D. P., H. Z., Y. H., H. S. and W. L. conducted the research; X. W., Y. L. and S. L. analysed the data; X. W. and Y. L. wrote the paper; Y. L. had primary responsibility for the final content. All authors read and approved the final manuscript.

The authors declare that they have no conflict of interest.

\section{References}

1. Blikslager AT, Moeser AJ, Gookin JL, et al. (2007) Restoration of barrier function in injured intestinal mucosa. Physiol Rev $87,545-564$.

2. Vilahur G, López-Bernal S, Camino S, et al. (2014) Lactobacillus plantarum CECT 7315/7316 intake modulates the acute and chronic innate inflammatory response. Eur J Nutr (Epublication ahead of print version 19 November 2014).

3. Liu Y, Huang J, Hou Y, et al. (2008) Dietary arginine supplementation alleviates intestinal mucosal disruption induced by Escherichia coli lipopolysaccharide in weaned pigs. Br J Nutr 100, 552-560.

4. Wang YJ, Liu W, Chen C, et al. (2013) Irradiation induced injury reduces energy metabolism in small intestine of Tibet minipigs. PLOS ONE 8, e58970.

5. Wu G (2013) Functional amino acids in nutrition and health. Amino Acids 45, 407-411.

6. Wang WW, Qiao SY \& Li DF (2009) Amino acids and gut function. Amino Acids 37, 105-110.

7. Wu G, Bazer FW, Davis TA, et al. (2007) Important roles for the arginine family of amino acids in swine nutrition and production. Livest Sci 112, 8-22.

8. Rhoads JM, Argenzio RA, Chen W, et al. (1995) Asparagine stimulates piglet intestinal $\mathrm{Cl}^{-}$secretion by a mechanism requiring a submucosal glutamate receptor and nitric oxide. J Pharmacol Exp Ther 274, 404-412.

9. Li P, Yin YL, Li D, et al. (2007) Amino acids and immune function. BrJ Nutr 98, 237-252.

10. Newburg DS, Frankel DL \& Fillios LC (1975) An asparagine requirement in young rats fed the dietary combinations of aspartic acid, glutamine and glutamic acid. J Nutr 105, 356-363.

11. McCormack SA, Tague LL, Gragoe EJ Jr, et al. (1990) Regulation of ornithine decarboxylase activity in LoVo cells. Am J Physiol 258, G934-G941.

12. Cantó C \& Auwerx J (2009) PGC-1 $\alpha$, SIRT1 and AMPK, an energy sensing network that controls energy expenditure. Curr Opin Lipidol 20, 98-105. 
13. Chau MD, Gao J, Yang Q, et al. (2010) Fibroblast growth factor 21 regulates energy metabolism by activating the AMPK-SIRT1-PGC-1 $\alpha$ pathway. Proc Natl Acad Sci U S A 107, 12553-12558.

14. Sanli T, Steinberg GR, Singh G, et al. (2014) AMP-activated protein kinase (AMPK) beyond metabolism: a novel genomic stress sensor participating in the DNA damage response pathway. Cancer Biol Ther 15, 156-169.

15. Wu SB \& Wei YH (2012) AMPK-mediated increase of glycolysis as an adaptive response to oxidative stress in human cells: implication of the cell survival in mitochondrial diseases. Biochim Biophys Acta 1822, 233-247.

16. Cantó C, Gerhart-Hines Z, Feige JN, et al. (2009) AMPK regulates energy expenditure by modulating $\mathrm{NAD}^{+}$metabolism and SIRT1 activity. Nature 458, 1056-1060.

17. Kim MY, Lim JH, Youn HH, et al. (2013) Resveratrol prevents renal lipotoxicity and inhibits mesangial cell glucotoxicity in a manner dependent on the AMPK-SIRT1-PGC1 $\alpha$ axis in $\mathrm{db} / \mathrm{db}$ mice. Diabetologia 56, 204-217.

18. Liu Y, Chen F, Odle J, et al. (2012) Fish oil enhances intestinal integrity and inhibits TLR4 and NOD2 signaling pathways in weaned pigs after LPS challenge. J Nutr 142, 2017-2024.

19. Puiman P \& Stoll B (2008) Animal models to study neonatal nutrition in humans. Curr Opin Clin Nutr Metab Care 11, 601-606.

20. Merrifield CA, Lewis M, Claus SP, et al. (2011) A metabolic system-wide characterisation of the pig: a model for human physiology. Mol Biosyst 7, 2577-2588.

21. NRC (1998) Nutrient Requirements of Swine, 10th ed. Washington, DC: National Academic Press.

22. Li S, Liu YL, Shi HF, et al. (2012) Effects of asparagine on growth performance, blood cell differential count and blood biochemical indices of weaned pigs challenged with lipopolysaccharide. Chinese J Anim Nutr 24, 2450-2458.

23. Hou Y, Yao K, Wang L, et al. (2011) Effects of $\alpha$-ketoglutarate on energy status in the intestinal mucosa of weaned piglets chronically challenged with lipopolysaccharide. $\mathrm{Br} J \mathrm{Nutr}$ 106, 357-363

24. Wu G \& Knabe DA (1994) Free and protein bound amino acids in sows colostrum and milk. J Nutr 124, 2437-2444.

25. Zhu HL, Liu YL, Xie XL, et al. (2013) Effect of L-arginine on intestinal mucosal immune barrier function in weaned pigs after Escherichia coli LPS challenge. Innate Immun 19, $242-252$

26. Lowry OH, Rosebrough NJ, Farr AL, et al. (1951) Protein measurement with folin phenol reagent. $J$ Biol Chem 193, $265-275$

27. Labarca C \& Paigen K (1980) A simple, rapid, and sensitive DNA assay procedure. Anal Biochem 102, 344-352.

28. Munro HN \& Fleck A (1969) Analysis of tissues and body fluids for nitrogenous constituents. In Mammalian Protein Metabolism, pp. 465-483 [HN Munro, editor]. New York, NY: Academic Press.

29. Liu Y, Han J, Huang J, et al. (2009) Dietary L-arginine supplementation improves intestinal function in weaned pigs after an Escherichia coli lipopolysaccharide challenge. Asian Aust J Anim Sci 22, 1667-1675.

30. Oliver WT \& Miles JR (2010) A low-fat liquid diet increases protein accretion and alters cellular signaling for protein synthesis in 10-day-old pigs. J Anim Sci 88, 2576-2584.

31. Weber TE, Trabue SL, Ziemer CJ, et al. (2010) Evaluation of elevated dietary corn fiber from corn germ meal in growing female pigs. J Anim Sci 88, 192-201.

32. Livak KJ \& Schmittgen TD (2001) Analysis of relative gene expression data using real-time quantitative PCR and $2^{-\Delta \Delta C_{\mathrm{T}}}$ method. Methods 25, 402-408.
33. Hou Y, Wang L, Zhang W, et al. (2012) Protective effects of $N$-acetylcysteine on intestinal functions of piglets challenged with lipopolysaccharide. Amino Acids 43, $1233-1242$.

34. Paszti-Gere E, Matis G, Farkas O, et al. (2014) The effects of intestinal LPS exposure on inflammatory responses in a porcine enterohepatic co-culture system. Inflammation 37, $247-260$.

35. Farkas O, Mátis G, Pászti-Gere E, et al. (2014) Effects of Lactobacillus plantarum 2142 and sodium $n$-butyrate in lipopolysaccharide-triggered inflammation: comparison of a porcine intestinal epithelial cell line and primary hepatocyte monocultures with a porcine enterohepatic co-culture system. J Anim Sci 92, 3835-3845.

36. Duarte S, Arango D, Parihar A, et al. (2013) Apigenin protects endothelial cells from lipopolysaccharide (LPS)induced inflammation by decreasing caspase- 3 activation and modulating mitochondrial function. Int J Mol Sci 14, 17664-17679.

37. Pinheiro DF, Pacheco PD, Alvarenga PV, et al. (2013) Maternal protein restriction affects gene expression and enzyme activity of intestinal disaccharidases in adult rat offspring. Braz J Med Biol Res 46, 287-292.

38. Rubio LA, Ruiz R, Peinado MJ, et al. (2010) Morphology and enzymatic activity of the small intestinal mucosa of Iberian pigs as compared with a lean pig strain. J Anim Sci 88, 3590-3597.

39. Blachier F, Boutry C, Bos C, et al. (2009) Metabolism and functions of L-glutamate in the epithelial cells of the small and large intestines. Am J Clin Nutr 90, 814S-821S.

40. Burrin DG \& Stoll B (2009) Metabolic fate and function of dietary glutamate in the gut. Am J Clin Nutr 90, 850S-856S.

41. Hardie DG \& Hawley SA (2001) AMP-activated protein kinase: the energy charge hypothesis revisited. Bioessays 23, 1112-1119.

42. Browne JL, Sanford PA \& Smyth DH (1977) Transport and metabolic processes in the small intestine. Proc $R$ Soc Lond B Biol Sci 195, 307-321.

43. Wiegand G \& Remington SJ (1986) Citrate synthase: structure, control, and mechanism. Annu Rev Biophys Biophys Chem 15, 97-117.

44. Corpas FJ, Barroso JB, Sandalio LM, et al. (1999) Peroxisomal NADP-dependent isocitrate dehydrogenase. Characterization and activity regulation during natural senescence. Plant Physiol 121, 921-928.

45. Bunik VI \& Strumilo S (2009) Regulation of catalysis within cellular network: metabolic and signaling implications of the 2-oxoglutarate oxidative decarboxylation. Curr Chem Biol 3, 279-290.

46. Sivakumar R, Anandh Babu PV \& Shyamaladevi CS (2008) Protective effect of aspartate and glutamate on cardiac mitochondrial function during myocardial infarction in experimental rats. Chem Biol Interact 176, 227-233.

47. Hardie DG (2003) Minireview: the AMP-activated protein kinase cascade: the key sensor of cellular energy status. Endocrinology 144, 5179-5183.

48. Takeuchi K, Morizane Y, Kamami-Levy C, et al. (2013) AMP-dependent kinase inhibits oxidative stress-induced caveolin-1 phosphorylation and endocytosis by suppressing the dissociation between c-Abl and Prdx1 proteins in endothelial cells. J Biol Chem 288, 20581-20591.

49. Fulco M, Cen Y, Zhao P, et al. (2008) Glucose restriction inhibits skeletal myoblast differentiation by activating SIRT1 through AMPK-mediated regulation of Nampt. Dev Cell 14, 661-673. 
50. Gerhart-Hines Z, Rodgers JT, Bare O, et al. (2007) Metabolic control of muscle mitochondrial function and fatty acid oxidation through SIRT1/PGC-1 $\alpha$. EMBO J 26, 1913-1923.

51. Gurd BJ (2011) Deacetylation of PGC- $1 \alpha$ by SIRT1: importance for skeletal muscle function and exerciseinduced mitochondrial biogenesis. Appl Physiol Nutr Metab 36, 589-597.
52. D'Errico I, Lo Sasso G, Salvatore L, et al. (2011) Bax is necessary for PGC1 $\alpha$ pro-apoptotic effect in colorectal cancer cells. Cell Cycle 10, 2937-2945.

53. Holecek M (2013) Side effects of long-term glutamine supplementation. JPEN J Parenter Enteral Nutr 37, 607-616.

54. Ren W, Chen S, Yin J, et al. (2014) Dietary arginine supplementation of mice alters the microbial population and activates intestinal innate immunity. J Nutr 144, 988-995. 